International Journal of

Environmental Research and

Public Health

ISSN 1660-4601

www.mdpi.com/journal/ijerph

Article

\title{
The Effects of Targeted Deliveries of Lovastatin and Tocotrienol on Ossification-Related Gene Expressions in Fracture Healing in an Osteoporosis Rat Model
}

\author{
Nurul 'Izzah Ibrahim ${ }^{\dagger}$, Norazlina Mohamed ${ }^{\dagger}$, Ima Nirwana Soelaiman ${ }^{\dagger}$ and \\ Ahmad Nazrun Shuid * \\ Department of Pharmacology, Faculty of Medicine, Universiti Kebangsaan Malaysia Medical Centre, \\ Jalan Yaacob Latif, Bandar Tun Razak, Cheras, 56000 Kuala Lumpur, Malaysia; \\ E-Mails: nurulizzah88@gmail.com (N.I.I.); azlina@ppukm.ukm.edu.my (N.M.); \\ imasoel@ppukm.ukm.edu.my (I.N.S.) \\ $\dagger$ These authors contributed equally to this work. \\ * Author to whom correspondence should be addressed; E-Mail: anazrun@yahoo.com; \\ Tel.: +60-3-9145-9576; Fax: +60-3-9145-9547.
}

Academic Editor: Paul B. Tchounwou

Received: 28 July 2015 / Accepted: 8 October 2015 / Published: 16 October 2015

\begin{abstract}
Osteoporotic drugs are used to prevent fragility fractures, but their role in fracture healing still remains unknown. Thus, alternative agents with suitable mode of delivery are needed to promote fracture healing. This study was performed to investigate the effects of direct deliveries of lovastatin and tocotrienol to fracture sites on ossification-related gene expression in fracture healing in a postmenopausal osteoporosis model. Forty-eight Sprague Dawley female rats were divided into six groups. Group I comprised the sham-operated rats, while Groups II-VI were ovariectomized rats. After 8 weeks, the right tibiae of all rats were fractured and stabilized. Group I and Group II were given two single injections of lovastatin and tocotrienol carriers. Group III was given an estrogen preparation at $64.5 \mu \mathrm{g} / \mathrm{kg}$ daily via oral gavages. Group IV was injected with lovastatin particles $(750 \mu \mathrm{g} / \mathrm{kg})$, while Group V was injected with tocotrienol particles $(60 \mathrm{mg} / \mathrm{kg})$. Group VI received two single injections of $750 \mu \mathrm{g} / \mathrm{kg}$ lovastatin particles and $60 \mathrm{mg} / \mathrm{kg}$ tocotrienol particles. After 4 weeks, the gene expressions were measured. Group VI showed significantly higher gene expressions of osteocalcin, BMP-2, VEGF- $\alpha$, and RUNX-2
\end{abstract}


compared to Group II. In conclusion, combined treatment of lovastatin and tocotrienol upregulated the expression of genes related to fracture healing.

Keywords: osteoporotic fracture healing; lovastatin; tocotrienol; targeted delivery; fracture healing genes

\section{Introduction}

Osteoporosis is a common skeletal disorder characterized by low bone mass and microarchitechural deterioration of bone tissue that predisposes patients to fragile bones and increased fracture risk [1]. Bone fragility and fractures due to osteoporosis can cause severe pain, disability, and in some cases, secondary complications that can even lead to death [2]. It is therefore important to speed up the fracture healing process to reduce the morbidity and socioeconomic costs associated with osteoporotic fractures.

Early diagnosis is essential for the management of osteoporosis. The most common diagnostic method is Dual-Energy X-ray Absorptiometry (DEXA), which has been validated in the clinical field for measuring bone density. However, DEXA is not suitable as a gold standard technique and as a screening tool in primary health care level for prevention purposes due to its radiation dose and high costs. Thus, a diagnostic method that uses ultrasound such as quantitative ultrasound scanner (QUS) which has a lower cost and no radiation exposure has become a more effective and reliable for assessing the quality of the bone [3]. The diagnostic sensitivity of QUS in the prediction of hip fracture has been shown to be similar to hip bone mineral density (BMD) measured with DEXA and superior to spine BMD [4]. However, QUS had lower specificity compared to DEXA, implicating that a lower T-score should be used ( $<3.65 \mathrm{SD})$ [5]. In addition to measure bone density, the osteoporosis assessment method for QUS can also provide information about the structure and elasticity of bones $[4,6]$.

The principal aim of osteoporosis management is to prevent fragility fractures by prescribing suitable anti-osteoporotic drugs such as estrogen replacement therapy and bisphosphonates. However, the effect of anti-osteoporotic drugs on bone fracture healing in humans is still not fully understood [7], and currently, no pharmacological treatments are available for fracture healing. There are reports that anti-osteoporotic drugs such as parathyroid hormone, bisphosphonates, and strontium renalate did not influence fracture healing [8]. Thus, there is a need to find suitable agents and new modes of delivery that may promote fracture healing of osteoporotic bone. Suitable agents could be from the commercial drugs or nutrients from natural products. The interactions between natural products and drugs may unintentionally reduce or increase the drug effect [9].

Statin, an anti-hyperlipidemic agent used to treat hypercholesterolemia had been found to exhibit anabolic actions on bone through the stimulation of BMP-2 expression [10]. The action of statin on bone formation was proposed by its activity on HMG-CoA reductase in mevalonate pathway, also known as cholesterol biosynthetic pathway [11]. Besides that, statin may also antagonize osteoclasts by increasing expression of osteoprotegrin in receptor activator of NF-Kb ligand (RANKL)-osteoprotegrin (OPG) pathway [12]. Lovastatin is a natural statin which can be used to treat various diseases including atherosclerosis, sepsis, peripheral arterial disease, ischemic disease and bone fracture [13]. 
Skoglund et al., reported that a high dose of statin given orally to femoral-fractured mice promoted fracture healing [14]. Statin given orally at a cholesterol-lowering dose does not reach the bones and only a minor fraction reached the fracture site [15], so a high oral dose of statin is required to achieve sufficient concentration at the bone fracture site. However, high doses of statin can cause adverse effects such as liver failure, kidney disease and rhabdomyolisis [16]. In order to get enough statin concentration at the fracture site using low dose of statin, it has to be delivered directly to the fracture site.

Tocotrienols are members of the vitamin E family, which is an essential nutrient that can be naturally sourced from palm, rice bran and annatto bean. The annatto bean-derived tocotrienols contain the most active types of tocotrienols; delta (90\%) and gamma tocotrienols (10\%) [17]. In an earlier study, a tocotrienol-enriched fraction showed improved fracture healing in a rat model when given orally, daily for 2 months [18]. The major intracellular transport protein for vitamin E is $\alpha$-tocopherol transfer protein. It is more selective towards $\alpha$-tocopherol than tocotrienols, resulting in shorter half-life and low bioavailability of the oral form of tocotrienol [19].

In order to improve the delivery of statin and tocotrienol to fracture sites, a suitable mode of delivery is needed. With a targeted delivery and a carrier system, a drug can primarily remain localized at the bone area to maintain sufficient concentration of the drug [20]. The purpose of this mode of delivery is to achieve a delivery profile that yields the desired drug level over a long period at the target site as the therapeutic agents are released at a constant rate [21]. With the limited amount of drug entering the systemic circulation, the drug side effects could be minimized [20]. Using targeted delivery system, the adverse effects associated with the use of high doses of lovastatin may be avoided, while the bioavailability of tocotrienol may be increased to promote fracture healing.

Fracture healing is a complex regenerative process in response to injury. It can be divided into reactive, healing and remodeling phases, which may last for four to six weeks. It involves intracellular and extracellular molecular signaling for bone induction and conduction [22,23]. During fracture healing process, bone is formed by two different processes; intramembranous ossification and endochondral ossification. Various bone matrix proteins and growth factors are recruited at the fracture injury site or from the circulation for the healing processes including osteocalcin, bone morphogenetic protein (BMP)-2, vascular endothelial growth factor (VEGF)- $\alpha$, bone sialoprotein, Runt-related transcription factor (Runx)-2 and FGF-23 [24-27]. Specifically, TGF- $\beta 2$ and - $\beta 3$ are released during intermediate phase for the proliferation of undifferentiated mesenchymal and progenitor cells, osteoblasts and chondrocytes $[22,28]$. Apart from that, the recruited cells also synthesize and secrete cartilage-specific matrix including Type II collagen [28]. All of these factors are responsible for stimulating chondrocyte proliferation, cartilage formation, osteoblast proliferation and bone synthesis [29]. Additionally, VEGF- $\alpha$ is important not only in osteogenesis but also in angiogenesis, to regulate blood vessel invasion during fracture healing [30].

To the best of our knowledge, the molecular mechanisms of osteoporotic fracture healing with the use of targeted deliveries of lovastatin and tocotrienol have not been elucidated. In this study, the fracture healing-related gene expressions were measured to understand the role of targeted delivery system of lovastatin and tocotrienol in promoting fracture healing. 


\section{Experimental Section}

\subsection{Animals and Surgical Procedures}

With the ethical approval obtained from Universiti Kebangsaan Malaysia Animal Ethical Committee (FP/FAR/2012/NAZRUN/21-NOV/472-NOV2012-MAR2014), forty-eight female Sprague Dawley rats, weighing 200 to $250 \mathrm{~g}$ were purchased from the laboratory animal resource unit. The rats were housed two per cage under 12-hour light-dark cycle and were given tap water ad libitum. Following acclimatization for 7 days, the rats were randomly divided into six groups. Group I comprised the sham-operated rats while Groups II, III, IV, V, VI were ovariectomized rats. The rats were ovariectomized to mimic a situation of estrogen-deficient state. Ovariectomized group rats (Groups II-VI) were left untreated for 8 weeks to allow for ovariectomy-induced osteoporosis. Fracture procedures were then performed according to a previous protocol described by Ibrahim et al. [31], in which pulsed ultrasound was used to fracture the right tibiae of all the rats and stabilized by means of a plate fixation method [32]. Intraperitoneal injection of ketamine and xylazil in 1:1 ratio was used to anaesthetize the animals. Then, the operated-side of the leg was shaved and sterilized with $70 \%$ alcohol, before incisions were performed. An anterior-medial approach with an extension from the medial femur condyle to the middle of the tibia was performed to gain access to the tibial bone. Then, without damaging the muscles at the tibia, the proximal tibial third was prepared in an epiperiosteal manner. A $90^{\circ}$ T-shaped titanium fixation plate XS (57-05140 Stryker Trauma, Selzach, Switzerland) with five holes was slightly pre-bent in the transversal part, and was fixed proximally with a $1.2 \times 4.0 \mathrm{~mm}$ screw to the anterior-medial surface of the tibia.

Osteotomy was performed at the metaphysis region using pulsed ultrasound (Piezosurgery, Mectron Medical Technology, Carasco, Italy). A complete fracture using the osteotomy method was confirmed visually. Following osteotomy, the plate was placed in its correct position and fixed with another $1.2 \times 4.0 \mathrm{~mm}$ screw distal to the anterior-medial surface of the tibia to immobilize the fracture. Instantly, two single injections of treatments $(0.05 \mathrm{~mL}$ per $100 \mathrm{~g}$ body weight $)$ were delivered into the muscles near the fracture site. Specific sites for injections of lovastatin particle or its carrier was located at $2 \mathrm{~mm}$ above the fracture line, while tocotrienol particle or its carrier was located at $2 \mathrm{~mm}$ below the fracture line. The incised skin was closed with non-absorbable suture. For post-operative care, each rat received injections of buprenorphine $(0.1 \mathrm{mg} / \mathrm{kg}$ of bodyweight every $12 \mathrm{~h}$ for 3 days $)$ as analgesic, baytril $5 \%(0.1 \mathrm{mg} / \mathrm{kg}$ body weight every $24 \mathrm{~h}$ for 5 days $)$ as antibiotic and iodine solution to the site as an antiseptic. Daily oral gavages of treatment were started one day after the fracture for a period of 4 weeks.

\subsection{Grouping of Rats and Treatments}

Both the Group I and Group II animals were given two single injections of lovastatin carrier and tocotrienol carrier. Group III was given daily oral gavages of Premarin $(64.5 \mu \mathrm{g} / \mathrm{kg})$ and two single injections of lovastatin carrier and tocotrienol carriers. Group IV was given two single injections of $750 \mu \mathrm{g} / \mathrm{kg}$ lovastatin particles and tocotrienol carrier. Group V was given two single injections of $60 \mathrm{mg} / \mathrm{kg}$ annatto tocotrienol particles and lovastatin carrier. Group VI was given two single injections of lovastatin particles and annatto tocotrienol particles at the same dose as the single 
treatment groups. All the groups were given daily oral gavages of deionised water, except for the Group III, which was given premarin and acted as positive control group. After four weeks of treatment period, the rats were euthanized by diethyl ether overdose. The fractured tibiae were dissected out and the plates and screws were removed. The tibiae were wrapped in gauze, immersed in phosphate buffered saline (PBS), and stored at $-70^{\circ} \mathrm{C}$ until analyzed.

\subsection{Preparation of Lovastatin and Tocotrienol Particles}

Lovastatin (catalog no. 1530, Tocris Bioscience, Bristol, UK) was used to prepare lovastatin particles as per an earlier protocol described by Ibrahim et al. [31]. An amount of $2.5 \mathrm{~mL}$ of $100 \mathrm{mg} / \mathrm{mL}$ poly-(D,L-lactide), viscosity of 0.26 to 0.54 (DURECT Corporation, Birmingham, AL, USA) was mixed with agitation with $1 \mathrm{~mL}$ of $50 \mathrm{mg} / \mathrm{mL}$ lovastatin in acetone. Then, with continuous stirring, $2.5 \mathrm{~mL}$ of water $1 \%$ polyvinyl alcohol (1\% PVA) was added in a drop wise manner. The mixture was stirred for $5 \mathrm{~h}$ in order to remove the remaining solvent and unloaded drugs. Following these, the mixture was centrifuged at 10,000/rpm and repeatedly washed with saline. After the centrifugation process, the sediment (resultant lovastatin particle) was collected and dried under reduced pressure overnight. Ultimately, the particles were resuspended in saline before injected to the rats.

Annatto tocotrienol (Delta Gold ${ }^{\circledR} 70$ ) was obtained from American River Nutrition Inc. (Hadley, MA, USA) for this study. The tocotrienol particles were prepared by solvent-evaporation method according to an earlier protocol described by Ibrahim et al. [31]. A total of $10 \mathrm{mg}$ of delta-tocotrienol and $50 \mathrm{mg}$ of poly-(D,L-lactide-co-glycolide) (PLGA) were dissolved in $2 \mathrm{~mL}$ of dichloromethane. Then, the solution was cooled to $4{ }^{\circ} \mathrm{C}$ for $1 \mathrm{~h}$, before being dispersed in $0.1 \%$ polyvinyl alcohol solutions. Stirring was performed continuously for $2 \mathrm{~h}$ at room temperature to remove the solvent. After that, the mixture was filtered using $0.45 \mu \mathrm{m}$ filter, washed with distilled water, and dried under reduced pressure for 2 days. Following these, the resultant tocotrienol particles were collected and dissolved in a solution of $0.5 \%$ carboxymethylcellulose (CMC) and $0.1 \%$ Tween 80 before administered to the rats. The same preparation methods were performed for lovastatin and tocotrienol carriers, but without the addition of lovastatin and tocotrienol, respectively.

\subsection{Measurement of Gene Expression}

Gene expressions were measured using the branched DNA technique (QuantiGene® 2.0 Plex Assay, Affymetrix Inc, Santa Clara, CA, USA). Tissue homogenates for gene expression measurement were prepared according to directions suggested by the manufacturer. Approximately $5 \mathrm{mg}$ samples (about the size of a rice grain) were taken from each tibia near the former fractured area and were placed in vial containing beads. Then, the samples were mixed with homogenizing solution and Proteinase $\mathrm{K}$ for tissue homogenates and the release of ribonucleic acid (RNA). The RNA samples were subjected in duplicate to Quantigene Plex assays to obtain the average for more accurate readings. The samples were hybridized overnight at $54{ }^{\circ} \mathrm{C}$ with specific mRNA capture beads and 2.0 capture probes. The following day, the samples were hybridized with 2.0 preamplifier and incubated with a 2.0 amplifier and a biotinylated label probe for $1 \mathrm{~h}$ at $50{ }^{\circ} \mathrm{C}$. This was followed by application of streptavidin-conjugated R-Phycoerythrin (SAPE) detection probe for $30 \mathrm{~min}$ at room temperature for fluorescence production. The fluorescent signals associated with individual capture 
beads were analyzed using a Bio-Plex 200 array reader (Luminex, Austin, TX, USA) equipped with Luminex 100 Xmap technology which measures the bead signature designating RNA target and the SAPE signal designating abundance. For each well, the total fluorescence from each individual bead type (corresponding to individual mRNA species) were subtracted with the average background fluorescence signals, and were then normalized to the fluorescence of a housekeeping gene. The housekeeping genes used in this study were glyceraldehyde-3-phosphate dehydrogenase (GAPDH), glucuronidase-beta (Gusb) and ribosomal protein S-18 (RPS-18). From the result of these three housekeeping genes, only one housekeeping gene that yields consistent average signal value was chosen. In this study, GAPDH was found to give consistent average values. The genes of interest are listed in Table 1.

Table 1. Genes of interest measured in this study.

\begin{tabular}{cccc}
\hline Gene Symbols & Other Symbols & Name of Genes & NCBI Accession Number \\
\hline BGLAP & BGP, BGPR, BGPRA & Osteocalcin & NM_013414 \\
BMP 2 & - & Bone morphogenetic proteins 2 & NM_017178 \\
VEGF $\alpha$ & VEGF & Vascular endothelial growth factor & NM_031836 \\
RUNX 2 & - & Runt-related transcription factor 2 & NM_346016 \\
FGF 23 & - & Fibroblast growth factor 23 & NM_130754 \\
TGF $\beta 2$ & - & Transforming growth factor beta 2 & NM_031131 \\
TGF $\beta 3$ & MGC 105479 & Transforming growth factor beta 3 & NM_013174 \\
\hline IBSP & BSP & Bone sialoprotein or & NM_012587 \\
\hline Col-2 $\alpha-1$ & CG2A1A,COLLII & Type II collagen & NM_012929 \\
\hline
\end{tabular}

\subsection{Statistical Analysis}

The data analysis was performed using Statistical Package for Social Science software (SPSS version 20.0, SPSS Inc., Armonk, NY, USA) and were expressed as mean \pm standard error mean (SEM). The data was tested for normality using Kolmogorov-Smirnov test. For normally distributed data, the statistical test used were the analysis of variance (ANOVA), followed by post-hoc analysis of Tukey's Honestly Significant Difference (HSD) test. The level of significance was taken as $p<0.05$.

\section{Results}

\subsection{Osteocalcin Gene Expression}

The osteocalcin gene expression of the tibial bones was significantly higher in Group VI compared to Group II $(p<0.05)$. There were no other significant differences in the osteocalcin gene expression levels among the various treatment groups (Figure 1). 


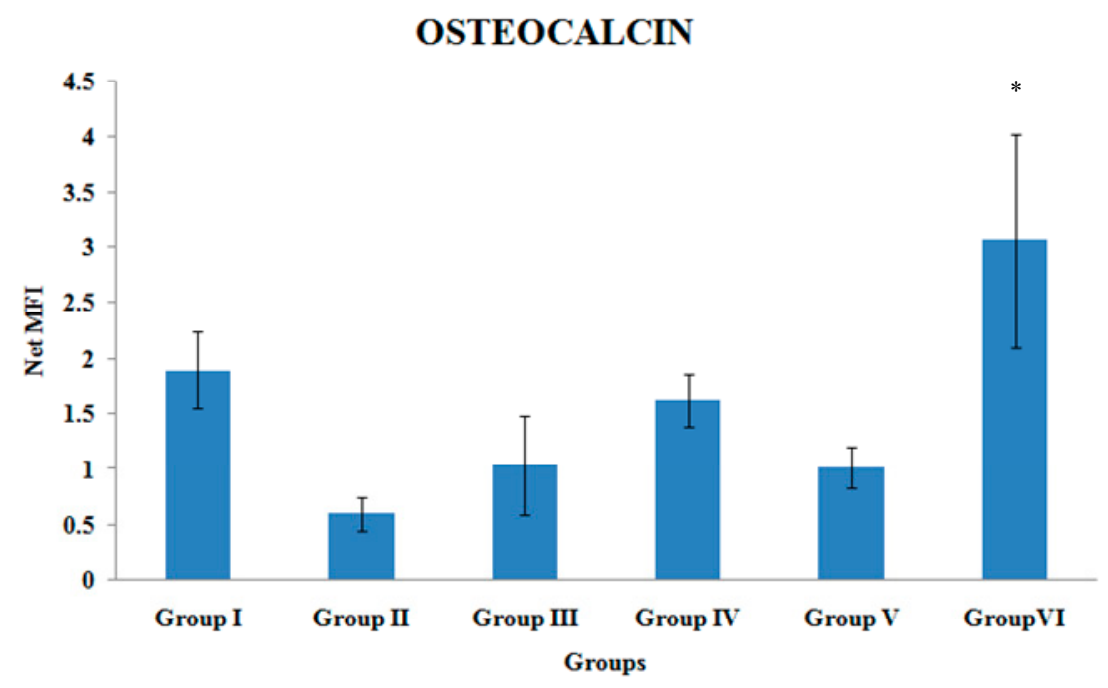

Figure 1. Osteocalcin gene expression. Samples were normalized to GAPDH MFI (Median Fluorescence Intensity). Values were expressed as mean \pm SEM. Group I: sham-operated group, Group II: ovariectomized control group, Group III: ovariectomized+Estrogen, Group IV: ovariectomized+Lovastatin, Group V: ovariectomized+Tocotrienol, Group VI: ovariectomized+Tocotrienol+Lovastatin. * Indicates a significant difference $(p<0.05)$ compared with the group II.

\subsection{BMP-2 Gene Expression}

The BMP-2 gene expression of the tibial bones was significantly higher in Group VI compared to Group II $(p<0.05)$. There were no other significant differences in the BMP-2 gene expression levels among the various treatment groups (Figure 2).

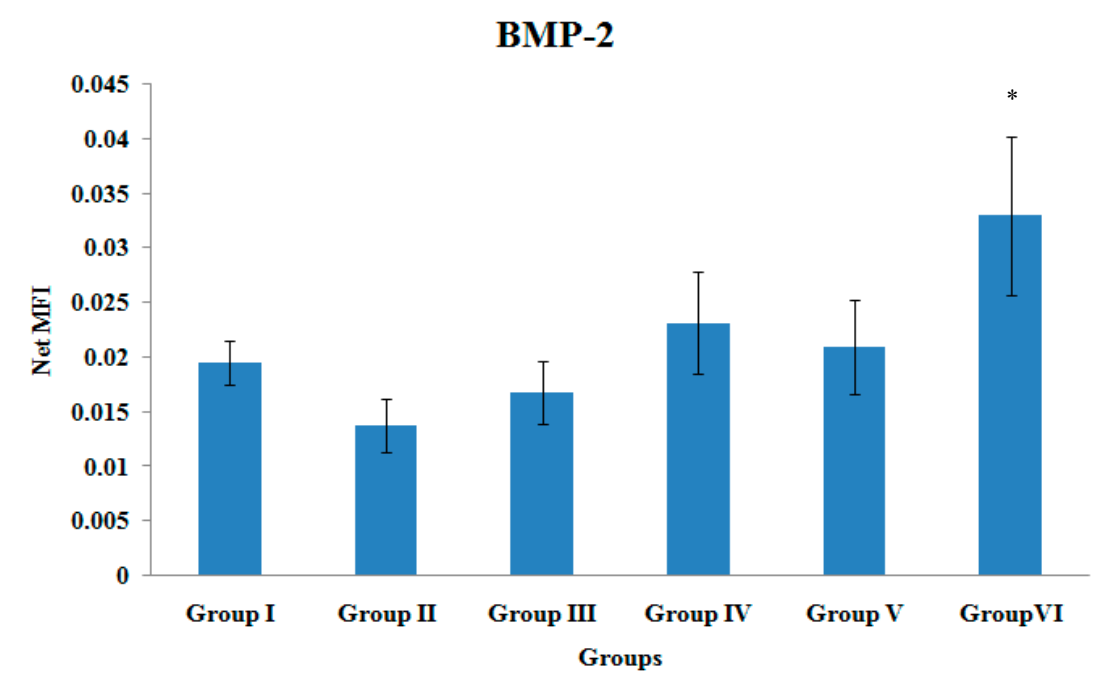

Figure 2. BMP-2 Gene Expression. Samples were normalized to GAPDH MFI (Median Fluorescence Intensity). Values were expressed as mean \pm SEM. Group I: sham-operated group, Group II: ovariectomized control group, Group III: ovariectomized+Estrogen, Group IV: ovariectomized+Lovastatin, Group V: ovariectomized+Tocotrienol, Group VI: ovariectomized+Tocotrienol+Lovastatin. * Indicates a significant difference $(p<0.05)$ compared with the group II. 


\subsection{VEGF- $\alpha$ Gene Expression}

The VEGF- $\alpha$ gene expression of the tibial bones was significantly higher in Group II, Group IV and Group VI when compared to Group II $(p<0.05)$. There were no other significant differences in the VEGF- $\alpha$ gene expression levels among the various treatment groups (Figure 3 ).

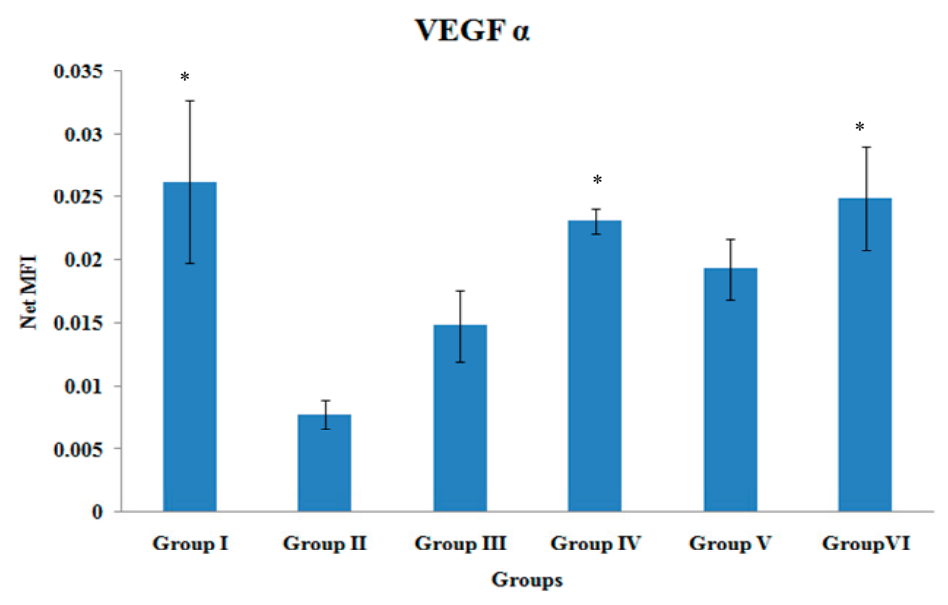

Figure 3. VEGF- $\alpha$ Gene Expression. Samples were normalized to GAPDH MFI (Median Fluorescence Intensity). Values were expressed as mean \pm SEM. Group I: sham-operated group, Group II: ovariectomized control group, Group III: ovariectomized+Estrogen, Group IV: ovariectomized+Lovastatin, Group V: ovariectomized+Tocotrienol, GroupVI: ovariectomized+Tocotrienol+Lovastatin. * Indicates a significant difference $(p<0.05)$ compared with the group II.

\subsection{RUNX-2 Gene Expression}

The Runx-2 gene expression of the tibial bones was significantly higher in Group VI when compared to Group II and Group III $(p<0.05)$. There were no other significant differences in the RUNX-2 gene expression levels among the various treatment groups (Figure 4).

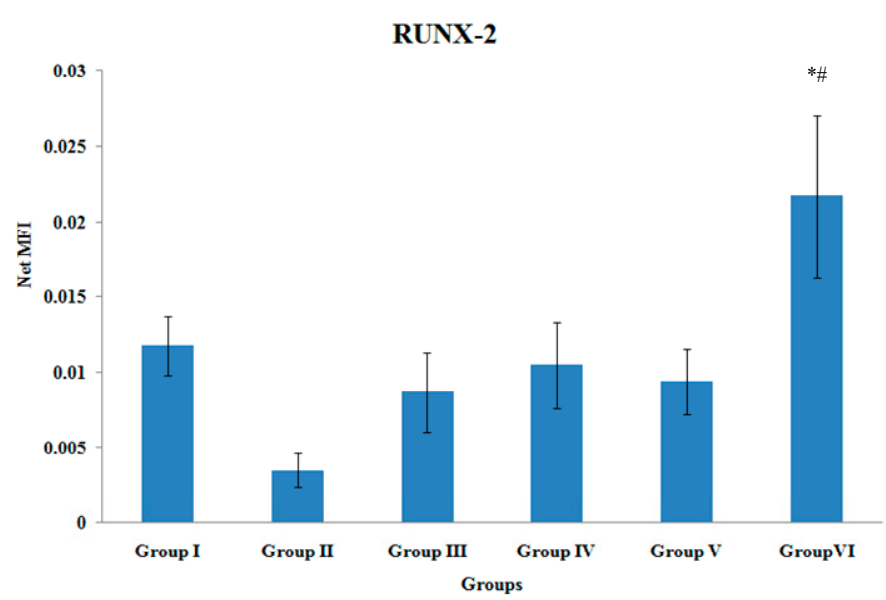

Figure 4. RUNX-2 Gene Expression. Samples were normalized to GAPDH MFI (Median Fluorescence Intensity). Values were expressed as mean \pm SEM. Group I: sham-operated group, Group II: ovariectomized control group, Group III: ovariectomized+Estrogen, Group IV: ovariectomized+Lovastatin, Group V: ovariectomized+Tocotrienol, Group VI: ovariectomized+Tocotrienol+Lovastatin. * Indicates a significant difference $(p<0.05)$ compared with the group II. ${ }^{\#}$ Indicates a significant difference $(p<0.05)$ compared with the group III. 


\subsection{BSP Gene Expression}

The BSP gene expression of the tibial bones was significantly higher in Group I when compared to Group II $(p<0.05)$. There were no other significant differences in the BSP gene expression levels among the various treatment groups (Figure 5).

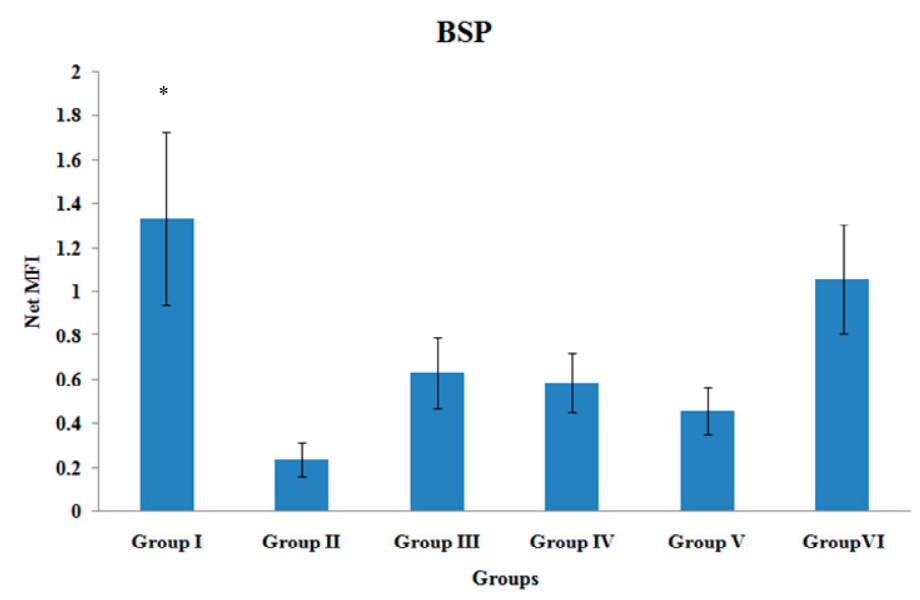

Figure 5. BSP Gene Expression. Samples were normalized to GAPDH MFI (Median FluorescenceIntensity). Values were expressed as mean \pm SEM. Group I: sham-operated group, Group II: ovariectomized control group, Group III: ovariectomized+Estrogen, Group IV: ovariectomized+Lovastatin, Group V: ovariectomized+Tocotrienol, Group VI: ovariectomized+Tocotrienol+Lovastatin. * Indicates a significant difference $(p<0.05)$ compared with the group II.

\subsection{TGF $\beta 2$ Gene Expression}

There were no significant differences in the TGF $\beta 2$ gene expression levels among the various treatment groups (Figure 6).

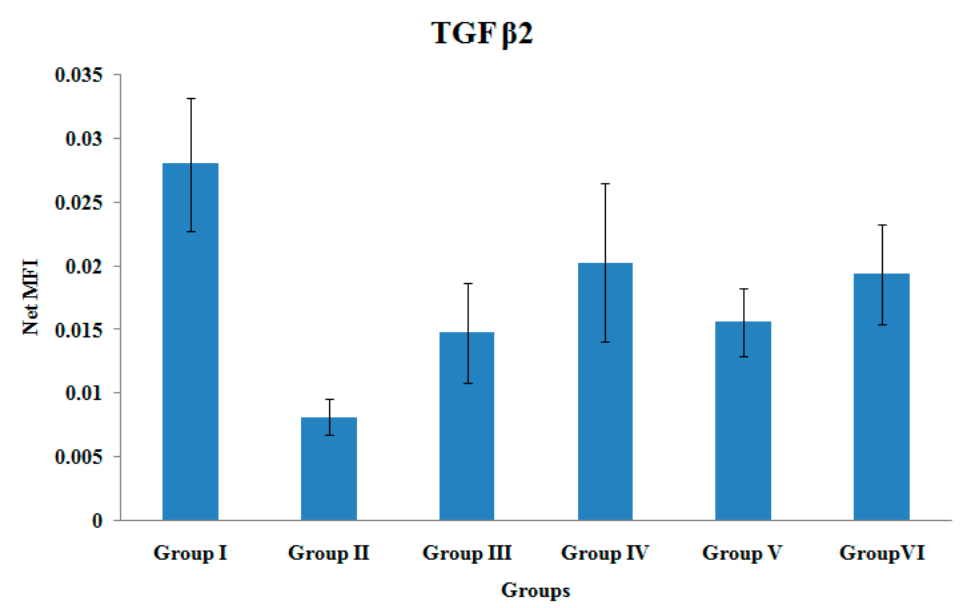

Figure 6. TGF $\beta 2$ Gene Expression. Samples were normalized to GAPDH MFI (Median Fluorescence Intensity). Values were expressed as mean \pm SEM. Group I: sham-operated group, Group II: ovariectomized control group, Group III: ovariectomized+Estrogen, Group IV: ovariectomized+Lovastatin, Group V: ovariectomized+Tocotrienol, GroupVI: ovariectomized +Tocotrienol+Lovastatin. 


\subsection{TGF $\beta 3$ Gene Expression}

There were no significant differences in the TGF $\beta 3$ gene expression levels among the various treatment groups (Figure 7).

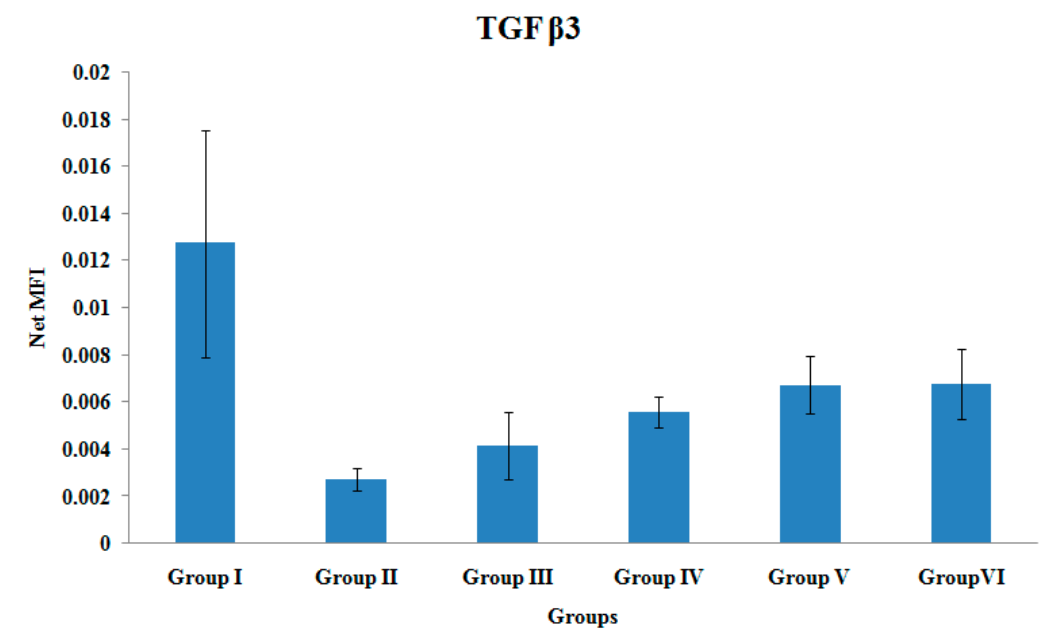

Figure 7. TGF $\beta 3$ Gene Expression. Samples were normalized to GAPDH MFI (Median Fluorescence Intensity). Values were expressed as mean \pm SEM. Group I: sham-operated group, Group II: ovariectomized control group, Group III: ovariectomized+Estrogen, Group IV: ovariectomized+Lovastatin, Group V: ovariectomized+Tocotrienol, Group VI: ovariectomized+Tocotrienol+Lovastatin.

\subsection{COL2 21 Gene Expression}

There were no significant differences in the COL2 $\alpha 1$ gene expression levels among the various treatment groups (Figure 8).

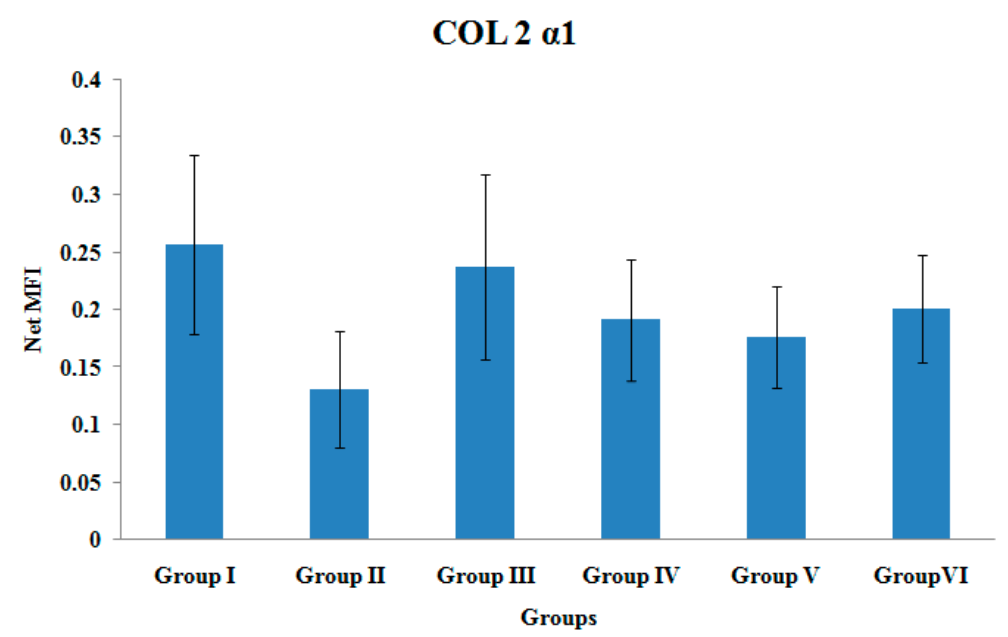

Figure 8. Col $2 \alpha 1$ Gene Expression. Samples were normalized to GAPDH MFI (Median Fluorescence Intensity). Values were expressed as mean \pm SEM. Group I: sham-operated group, Group II: ovariectomized control group, Group III: ovariectomized+Estrogen, Group IV: ovariectomized+Lovastatin, Group V: ovariectomized+Tocotrienol, Group VI: ovariectomized+Tocotrienol+Lovastatin. 


\subsection{FGF-23 Gene Expression}

There were no significant differences in the FGF-23 gene expression levels among the various treatment groups (Figure 9).

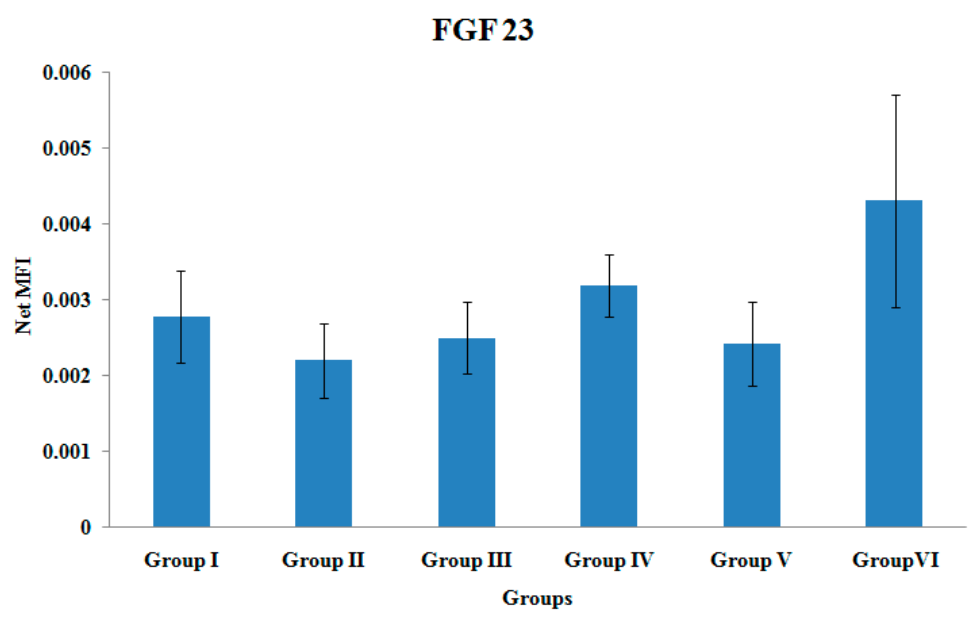

Figure 9. FGF 23 Gene Expression. Samples were normalized to GAPDH MFI (Median Fluorescence Intensity). Values were expressed as mean \pm SEM. Group I: sham-operated group, Group II: ovariectomized control group, Group III: ovariectomized+Estrogen, Group IV: ovariectomized+Lovastatin, Group V: ovariectomized+Tocotrienol, Group VI: ovariectomized+Tocotrienol+Lovastatin .

\section{Discussion}

In this present study, gene expression was measured at one-time point (4 weeks post-fracture), which was referred to as intermediate phase of fracture healing in rats [31]. This phase consisted of fibrocartilage callus formation and bony callus formation. The genes measured in this study were selected based on their important roles during the intermediate phase of fracture healing. Type II collagen was chosen as it is a major structural protein of cartilage, which appear during fibrocartilage callus formation of intermediate phase. TGF- $\beta 2$, TGF- $\beta 3$, BMP-2, and osteocalcin were important for bony callus formation during the intermediate phase [24]. Apart from that, VEGF is crucial for angiogenesis during fracture healing [30].

The result of this present study showed that combined targeted deliveries of tocotrienol and lovastatin (Group VI) significantly elevated the gene expressions of BMP-2, VEGF- $\alpha$, osteocalcin, and Runx-2 when compared to the ovariectomized-control rats (Group II). The up-regulation of these fracture healing-related gene expressions might be related to the suppression of HMG-CoA reductase on the mevalonate pathway (Figure 10). The findings of this present study were consistent with a previous study by Abdul-Majeed et al., which reported that combined oral administration of tocotrienol and lovastatin significantly increased bone formation and reduced bone resorption of ovariectomy-induced osteoporotic rats [33]. On the other hand, other studies showed that high oral doses of statin are required to enhance bone formation and reduced bone resorption in rodents, when statin was used as a single agent $[10,34,35]$. These high oral doses of statin were associated with myotoxicity and hepatotoxicity in human [36,37]. Abdul-Majeed et al., overcame this problem by 
combining an oral statin at clinically acceptable dose with tocotrienol to achieve both bone anti-osteoporotic and anabolic activities. These agents may have exhibited additive or synergistic effect to increase bone formation of osteoporotic rats [33]. However, it is difficult to get high and constant levels of statins in the bone due to their limited distribution to the peripheral tissues after oral administration [15]. The distribution of oral tocotrienol was also poor due to poor selectivity by the $\alpha$-tocopherol transfer protein at the liver [19]. Both of these problems could be avoided by administrating the agents directly to the bone using a targeted delivery system.

The present study showed that these agents were effective in improving osteoporotic fracture healing when administered with a targeted delivery system. Earlier result of combined targeted deliveries of tocotrienol and lovastatin showed improvement in mineralization and strength of callus formed during fracture healing of osteoporotic bone [31]. Using this delivery system, low doses of the agents are combined with their suitable carriers and they are released slowly with a single injection to maintain high concentrations at the bone microenvironment. Both lovastatin and tocotrienol have similar activity on HMG-CoA reductase in mevalonate pathway. However, the mechanisms are different. Statin act as a chemical analogue of substrate HMG-CoA and block the activity of HMG-CoA reductase [38]. Meanwhile, for tocotrienol, the inhibition of HMG-CoA reductase activity is regulated post-transcriptionally through the increased cellular conversion of farnesyl pyrophosphate to farnesol $[39,40]$. These could upregulate BMP-2 gene expression by decreasing protein prenylation of isoprenoids intermediates, such as farnesyl pyrophosphate (FPP) and geranylgeranyl pyrophosphate (GGPP) (Figure 10) [11,41]. The decreased protein prenylation caused a reduction of RhoA, which is a prenylated protein and resulted into increased BMP-2 gene expression. Hagihara et al., have confirmed that the inhibition of RhoA was able to promote BMP-2 induced osteoblastic differentiation processes [42]. These actions may have resulted in the significantly higher expression of BMP-2 genes for the combination group (Group VI) found in this study.

Moreover, in this study, the combination group had also recorded a significantly higher osteocalcin gene expression. This increased expression may have occurred following the BMP-2 induced osteoblastic differentiation processes. Osteoblast cells secrete osteoid and synthesize osteocalcin during bone formation, which generally serves as a specific marker for osteoblast activity and bone formation [43].

In the present study, osteocalcin gene expression was measured 4 weeks after the induction of fracture in rats, which corresponded with the reparative phase of fracture healing. The combination group (Group VI) showed significantly higher osteocalcin gene expression compared to the ovariectomized control group (Group II). This was consistent with the findings by Yao et al., where osteocalcin was expressed later and was elevated after the 28th day of culture period [44]. Moreover, Jingushi et al., reported that osteocalcin gene expression was detected only in the hard callus during the later healing stages of endochondoral ossification and remodeling. It was not expressed in soft callus during intramembranous ossification stage [45]. All these findings were consistent with the present study, which showed that osteocalcin was highly expressed by the combination group (Group VI) during the reparative phase. This indicates better fracture healing with the highest activity of bone formation when tocotrienol and statin were delivered together to the fracture site. 


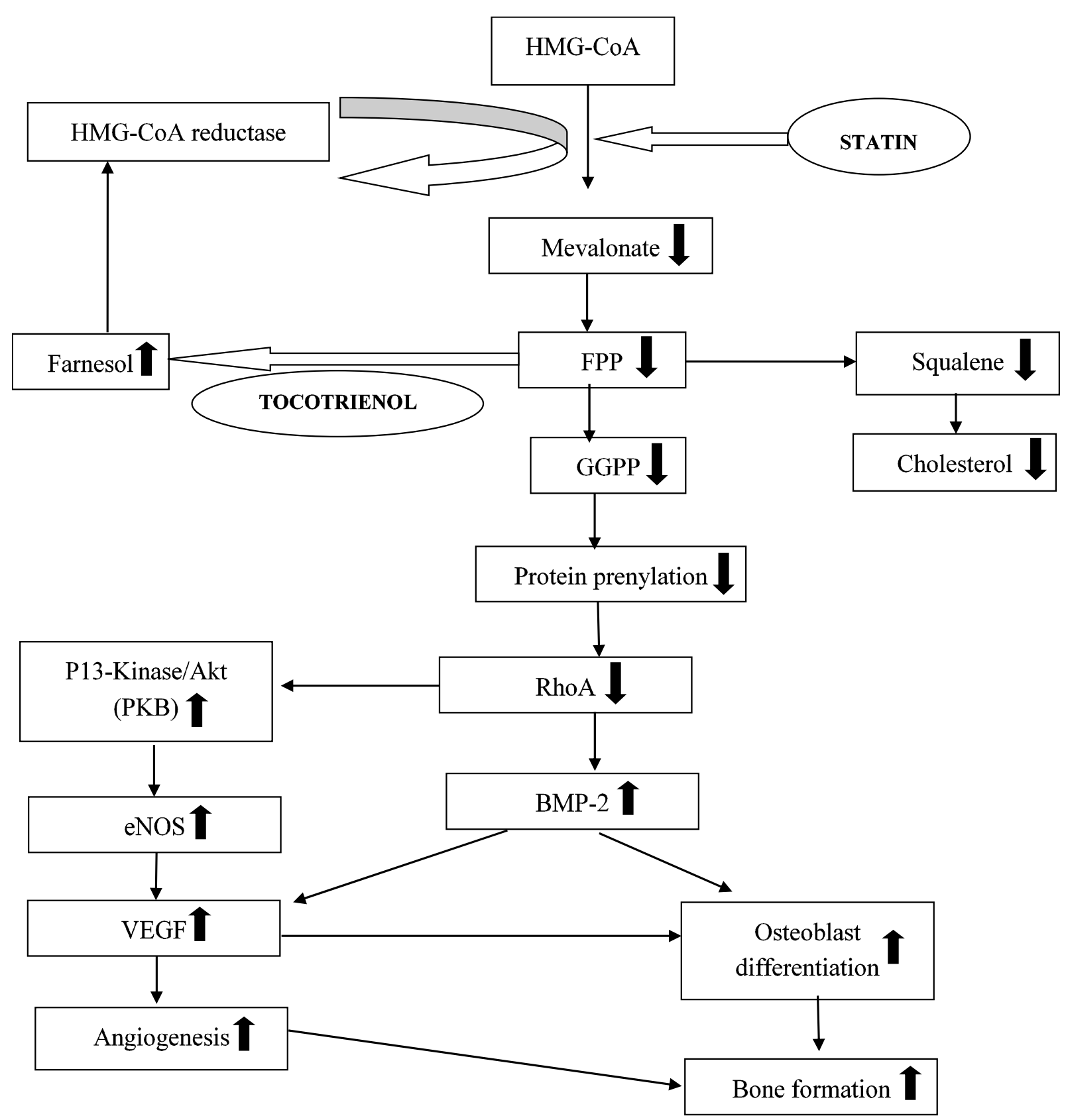

Figure 10. The different mechanisms of tocotrienol and statin to suppress the activity of HMG-CoA reductase. The figure was modified from Ibrahim et al. 2013 [41].

In this present study, Group VI showed significantly higher RUNX-2 gene expression, which might also be correlated with the high BMP-2 gene expression. This result is consistent with Phimphilai et al., who found that BMP signaling is required to stimulate expressions of many target genes including RUNX2. When the BMP signaling was inhibited, RUNX-2 failed to stimulate osteoblast differentiation as it was not able to transactivate the osteocalcin gene promoter-luciferase reporter [46]. Interestingly, the RUNX-2 gene expression of Group VI was significantly higher than Group III (estrogen treatment group). The estrogen group, which acted as the positive control group in the current study, did not show any significant changes on all the gene expressions measured. This was not expected but Cao et al. [47] reported that estrogen treatment failed to promote femoral fracture healing of osteoporotic rats. In contrast, Estai et al. [48] reported estrogen-promoting effects on the 
healing of femoral fracture in osteoporotic rats as shown by the enhanced strength of healed bone. It is an accepted fact that estrogen prevents bone loss in postmenopausal women by suppressing bone resorption activity [49]. However, estrogen may cause mild suppression of bone formation activity, which may negatively affect bone remodeling and fracture healing [47,50]. All these factors could be the reasons behind the insignificant effects of estrogen on fracture healing.

In the present study, Group VI showed significant higher VEGF- $\alpha$ gene expression compared to Group II. VEGF gene expression is required in fracture healing for promotion of vasculogenesis and angiogenesis. These allowed deliveries of osteogenic substrates, pericyte stem cells, and mesenchymal stem cells for differentiation of osteoblast and bone formation [51,52]. The stimulation of VEGF gene expression during fracture healing was also closely related to BMP-2 gene expression. It was reported that BMP-2 is capable of stimulating murine-derived angiogenesis in fetal mice bone [53]. Maeda et al., documented that statins could stimulate VEGF expression. This was consistent with our study as the VEGF gene expression was significantly elevated in the rats receiving lovastatin (Group IV). The VEGF stimulation occurred via activation of the P13K/Akt pathway to promote osteoblast differentiation [54].

There were no significant differences in the gene expressions of TGF- $\beta_{2}$, and TGF- $\beta_{3}$. Cho et al., have demonstrated that TGF- $\beta$ superfamily such as GDF-5, TGF- $\beta_{2}$, and TGF- $\beta_{3}$ showed maximal expressions on day 7 after fracture [22]. On the other hand, Abe et al., measured gene expressions in murine bone marrow cultures from adult mice and reported that TGF- $\beta_{2}$ and TGF- $\beta_{3}$ were expressed from day 3 (week 1) to day 21 (week 3) [55]. TGF- $\beta_{2}$ and TGF- $\beta_{3}$ were expressed early to initiate signaling for BMP synthesis by osteoprogenitor cells, which is important during the early phase of fracture healing [56]. The TGF- $\beta$ gene expressions may have been low when their levels were measured by the fourth week of fracture healing in the present study.

FGF23 is a bone-derived hormone that regulates phosphorus and vitamin D metabolism. It has recently been suggested as a putative marker of bone healing [57]. In the current study, Group VI showed higher FGF23 gene expression than other groups but did not reach statistical significance. Goebel et al., reported a marked increase in FGF23 mRNA expression in ovine model with normal fracture healing compared to those with delayed course of healing. This showed that the elevated levels of FGF 23 are associated with uneventful fracture healing and can act as an indicator for healing prone to reunion versus nonunion [27]. Thus, the highly expressed FGF23 gene in Group VI in this current study may indicate uncomplicated fracture healing.

Bone sialoprotein (BSP) gene expression during fracture healing is important for biomineralization of connective tissues [58]. Group VI showed elevated BSP gene expression although it did not reach significant value when compared to Group II. Schmid et al., reported maximal expression of BSP at 14 days after tibial fracture in mice [26]. Meanwhile, Chen et al., reported that during embryogenesis and growth of rat tissues, BSP mRNA transcripts were first evident in fully differentiated osteoblast, with maximal expression observed at 21 days gestation [58]. These studies showed that BSP expression has a specific role in mediating the initial stages of tissue mineralization.

Gene expression of type II collagen was measured as an index of matrix synthesis of cartilage [59]. Hatano et al., reported that mevastatin, another type of statin, was able to increase type II collagen mRNAs at day 2 on rat-cultured chondrocytes. However, at longer period of time (10 days), type II collagen mRNAs was decreased with mevastatin treatment [60]. This study showed that statin 
increased type II collagen during early treatment period. Apart from that, Jingushi et al., also reported similar result, where type II collagen gene was maximally expressed during chondrogenesis, specifically after nine days of fracture [45]. Meyer et al., reported that type II collagen mRNA expression was decreased at fourth week of fracture healing and was non-detectable at sixth week of fracture healing [59]. These studies showed that type II collagen gene expression was highly expressed during the early phase of fracture healing, which could be the reason why no significant change was detected in its expression in the present study.

\section{Conclusions}

In conclusion, lovastatin and tocotrienol particles administered directly to the fracture site using a controlled drug delivery system, could up-regulate crucial genes for fracture healing such as osteocalcin, BMP-2, VEGF- $\alpha$ and RUNX-2. Single treatment with lovastatin using the same delivery system appeared to affect VEGF gene expression only. Therefore, combined targeted delivery of lovastatin and tocotrienol may have the ability to promote fracture healing. For future prospective osteoporotic fracture healing studies, a time-point measurement of gene expression should be performed to make sure all genes of interest can be measured at their maximal expression.

\section{Acknowledgments}

The authors would like to thank the Universiti Kebangsaan Malaysia (UKM), the Ministry of Higher Education for the grant (ERGS/1/2012/SKK03/UKM/02/1) and the Pharmacology Department staffs for their technical support. We would also like to acknowledge American River Nutrition Inc. for supplying annatto tocotrienol.

\section{Author Contributions}

Nurul 'Izzah Ibrahim carried out the gene expression studies, performed the statistical analysis and wrote the manuscript. Ahmad Nazrun Shuid participated in the study design and development and helped to draft and revises the manuscript. Ima Nirwana Soelaiman and Norazlina Mohamed involved in designing the research. All authors read and approved the final manuscript.

\section{Conflicts of Interest}

The authors declared to have no conflict of interests. The authors are responsible for the content and writing of this paper.

\section{References}

1. National Osteoporosis Foundation. Clinician's Guide to Prevention and Treatment of Osteoporosis; National Osteoporosis Foundation: Washington, DC, USA, 2010.

2. Johnell, O.; Kanis, J.A. An estimate of the worldwide prevalence and disability associated with osteoporotic fractures. Osteoporos. Int. 2006, 17, 1726-1733. 
3. Pisani, P.; Renna, M.D.; Conversano, F.; Casciaro, E.; Muratore, M.; Quarta, E.; Paola, M.D.; Casciaro, S. Screening and early diagnosis of osteoporosis through X-ray and ultrasound based techniques. World J. Radiol. 2013, 5, 398-410.

4. Njeh, C.F.; Boivin, C.M.; Langton, C.M. The role of ultrasound in the assessment of osteoporosis: A review. Osteoporos. Int. 1997, 7, 7-22.

5. Trimpou, P.; Bosaeus, I.; Bengtsson, B.; Landin-Wilhemsen, K. High correlation between quantitative ultrasound and DXA during 7 years follow-up. Eur. J. Radiol. 2010, 73, 360-364.

6. Fuerst, T.; Glüer, C.C.; Genant, H.K. Quantitative ultrasound. Eur. J. Radiol. 1995, 20, 188-192.

7. Alegre, D.N.; Ribeiro, C.; Sousa, C.; Correia, J.; Silva, L.; de Almeida, L. Possible benefits of strontium ranelate in complicated long bone fractures. Rheumatol. Int. 2012, 32, 439-443.

8. Jørgensen, N.R.; Schwarz, P. Effects of anti-osteoporosis medications on fracture healing. Curr. Osteoporos. Rep. 2011, 9, 149-155.

9. Bushra, R.; Aslam, N.; Khan, A.Y. Food-drug interactions. Oman Med. J. 2011, 26, 77-83.

10. Mundy, G.; Garrett, R.; Harris, S.; Chan, J.; Chen, D.; Rossini, G.; Boyce, B.; Zhao, M.; Gutierrez, G. Stimulation of bone formation in vitro and in rodents by statins. Science 1999, 286, 1946-1949.

11. Horiuchi, N.; Maeda, T. Statins and bone metabolism. Oral Dis. 2006, 12, 85-101.

12. Tsartsalis, A.N.; Dokos, C.; Kaiafa, G.D.; Tsartsalis, D.N.; Kattamis, A.; Hatzitolios, A.I.; Savopoulous, C.G. Statins, bone formation and osteoporosis: Hope or hype? Hormones 2012, 11, 126-139.

13. Seraman, S.; Rajendran, A.; Thangavelu, V. Statistical optimization of anticholesterolemic drug lovastatin production by the red mold Monascus purpureus. Food Bioprod. Process. 2010, 88, 266-276.

14. Skoglund, B.; Forslund, C.; Aspenberg, P. Simvastatin improves fracture healing in mice. J. Bone Miner. Res. 2002, 17, 2004-2008.

15. Hamelin, B.A.; Turgeon, J. Hydrophilicity/lipophilicity: Relevance for the pharmacology and clinical effects of HMG-CoA reductase inhibitors. Trends Pharmacol. Sci. 1998, 19, 26-37.

16. Guyton, J.R. Benefits versus risk in statin treatment. Am. J. Cardiol. 2006, 97, 95-97.

17. Tan, B. Tocotrienols: The New Vitamin E. Spacedocnet. 2010. Available online: http://www.spacedoc.net (accessed on 15 July 2015).

18. Sharlina, M.; Nazrun, A.S.; Mokhtar, S.A.; Abdullah, S.; Soelaiman, I.N. Tocotrienol supplement improves late phase healing compared to alpha tocopherol in a rat model of Postmenopausal osteoporosis: A biomechanical Evaluation. Evid. Based Complement. Altern. 2012, 2012, doi:10.1155/2012/372878.

19. Packer, L.; Weber, S.U.; Rimbach, G. Molecular aspects of $\alpha$-tocotrienol antioxidant action and cell signaling. J. Nutr. 2001, 131, 369S-373S.

20. Shuid, A.N.; Ibrahim, N.I.; Mohd Amin, M.C.I.; Mohamed, I.S. Drug delivery systems for Prevention and treatment of osteoporotic fracture. Curr. Drug Targets 2013, 14, 1558-1564.

21. Shaik, M.R.; Korsapati, M.; Panati, D. Polymers in controlled drug delivery systems. Int. J. Pharma Sci. 2012, 2, 112-116. 
22. Cho, T.J.; Gerstenfeld, L.C.; Einhorn, T.A. Differential temporal expression of members of the transforming growth factor beta superfamily during murine fracture healing. J. Bone Miner. Res. 2002, 17, 513-520.

23. Einhorn, T.A. The cell and molecular biology of fracture healing. Clin. Orthop. 1998, 355, 7-21.

24. Sfeir, C.; Ho, L.; Doll, B.A.; Azari, K.; Hollinger, J.O. Fracture repair. In Bone Regeneration and Repair: Biological and Clinical Applications; Lieberman, J.R., Friedlaender, G.E., Eds.; Human Press Inc.: Totowa, NJ, USA, 2005; p. 22.

25. Ai-Aql, Z.S.; Alagl, A.S.; Graves, D.T.; Gerstenfeld, L.C.; Einhorn, T.A. Molecular mechanisms controlling bone formation during fracture healing and distraction osteogenesis. J. Dent. Res. 2008, 87, 107-118.

26. Schmid, G.J.; Kobayashi, C.; Sandell, L.J.; Ornitz, D.M. Fibroblast growth factor expression during skeletal fracture healing in mice. Dev. Dyn. 2009, 238, 766-774.

27. Goebel, S.; Lienau, J.; Rammoser, U.; Seefried, L.; Wintgens, K.F.; Seufert, J.; Duda, G.; Jakob, F.; Ebert, R. FGF23 is a putative marker for bone healing and regeneration. J. Orthop. Res. 2009, 27, 1141-1146.

28. Tsiridis, E.; Upadhyay, N.; Giannoudis, P. Molecular aspects of fracture healing: Which are the important molecules? Injury 2007, 38, S11-S25.

29. Doll, B. Gene expression during fracture repair. In Musculoskeletal Regeneration: Biological Materials and Methods; Pietrzak, W.S., Ed.; Human Press Inc.: Totowa, NJ, USA, 2008; p. 46.

30. Yang, Y.Q.; Tan, Y.Y.; Wong, R. The role of vascular endothelial growth factor in ossification. Int. J. Oral Sci. 2012, 4, 64-68.

31. Ibrahim, N.; Khamis, M.F.; Mod Yunoh, M.F.; Abdullah, S.; Mohamed, N.; Shuid, A.N. Targeted delivery of lovastatin and tocotrienol to fracture site promotes fracture healing in osteoporosis model: Micro-computed Tomography and Biomechanical Evaluation. PLoS ONE 2014, 9, doi:10.1371/journal.pone.0115595.

32. Stuermer, E.K.; Sehmisch, S.; Rack, T.; Wenda, E.; Seidlova-Wuttke, D.; Tezval, M.; Wuttke, W.; Frosch, K.H.; Stuermer, K.M. Estrogen and reloxifene improve metaphyseal fracture healing in the early phase of osteoporosis. A new fracture-healing model at the tibia in rat. Langerbecks Arch. Surg. 2010, 395, 163-172.

33. Abdul-Majeed, S.; Mohamed, N.; Soelaiman, I.N. Effects of Tocotrienol and Lovastatin Combination on Osteoblast and Osteoclast Activity in Estrogen-Deficient Osteoporosis. Evid. Based Complement. Altern. Med. 2012, 2012, 1-9.

34. Maritz, F.J.; Conradie, M.M.; Hulley, P.A.; Gopal, R.; Hough, S. Effect of statins on bone mineral density and bone histomorphometry in rodents. Arterioscler. Thromb. Vasc. Biol. 2001, 21, 1636-1641.

35. Ho, M.L.; Chen, Y.H.; Liao, H.J.; Chen, C.-H.; Hung, S.-H.; Lee, M.-J.; Fu, Y.-C.; Wang, Y.-H.; Wang, G.-J.; Chang, J.-K. Simvastatin increases osteoblasts and osteogenic proteins in ovariectomized rats. Eur. J. Clin. Investig. 2009, 39, 296-303.

36. Jacobson, R.H.; Wang, P.; Glueck, C.J. Myositis and rhabdomyolysis associated with concurrent use of simvastatin and nefazodone. J. Am. Med. Assoc. 1997, 277, 296-297.

37. Fuentes, I.; Aguilera, C. Myopathy secondary to the treatment with inhibitors of HMG-CoA reductase. Med. Clin. 1998, 111, 700-704. 
38. Correll, C.C.; Edwards, P.A. Mevalonic acid-dependent degradation of 3-hydroxy-3methylglutaryl-coenzyme a reductase in vivo and in vitro. J. Biol. Chem. 1994, 269, 633-638.

39. Parker, R.A.; Pearce, B.C.; Clark, R.W.; Gordon, D.A.; Wright, J.J. Tocotrienols regulate cholesterol production in mammalian cells by post-transcriptional suppression of 3-hydroxy-3-methylglutaryl-coenzyme A reductase. J. Biol. Chem. 1993, 268, 11230-11238.

40. Song, B.L.; DeBose-Boyd, R.A. Insig-dependent ubiquitination and degradation of 3-hydroxy-3-methylglutaryl coenzyme A reductase stimulated by $\delta$ - and $\gamma$-tocotrienols. $J$. Biol. Chem. 2006, 281, 25054-25061.

41. Ibrahim, N.; Mohamed, N.; Shuid, A.N. Update on statins: Hope for osteoporotic fracture healing treatment. Curr. Drug Targets 2013, 14, 1524-1532.

42. Hagihara, M.; Endo, M.; Hata, K. Neogenin, a receptor for bone morphogenetic proteins. J. Biol. Chem. 2011, 286, 5157-5165.

43. Dogan, E.; Posaci, C. Monitoring hormone replacement therapy by biochemical markers of bone metabolism in menopausal women. Postgrad. Med. J. 2002, 78, 727-731.

44. Yao, K.L.; Todescan, R.; Sodek, J. Temporal changes in matrix protein synthesis and mRNA expression during mineralized tissue formation by adult rat bone marrow cells in culture. $J$. Bone Miner. Res. 1994, 9, 231-240.

45. Jingushi, S.; Joyce, M.E.; Bolander, M.E. Genetic expression of extracelluar matrix proteins correlates with histologic changes during fracture repair. J. Bone Miner. Res. 1992, 7, 1045-1055.

46. Phimphilai, M.; Zhao, Z.; Boules, H.; Roca, H.; Franceschi, R.T. BMP Signaling Is Required for RUNX2-Dependent Induction of the Osteoblast Phenotype. J. Bone Miner. Res. 2006, 21, 637-646.

47. Cao, Y.; Mori, S.; Mashiba, T.; Westmore, M.S.; Ma, L.; Sato, M.; Akiyama, T.; Shi, L.; Komatsubara, S.; Miyamoto, K.; et al. Raloxifene, estrogen, and alendronate affect the processes of fracture repair differently in ovariectomized rats. J. Bone Miner. Res. 2002, 17, 2237-2246.

48. Estai, M.A.; Suhaimi, F.H.; Das, S.; Shuid, A.N.; Zahiah Mohamed, Z.; Soelaiman, I.N.; Expression of TGF- $\beta 1$ in the blood during fracture repair in an estrogen-deficient rat model. Clinics 2011, 66, 2113-2119.

49. Cauley, J.A.; Seeley, D.G.; Ensrud, K.; Ettinger, B.; Black, D.; Cummings, S.R.; Study of Osteoporotic Fractures Research Group. Estrogen replacement therapy and fractures in older women. Ann. Intern. Med. 1996, 122, 9-16.

50. Sato, M.; Bryant, H.U.; Iversen, P.; Helterbrand, J.; Smietana, F.; Bemis, K.; Higgs, R.; Turner, C.H.; Owan, I.; Takano, Y.; et al. Advantages of raloxifene over alendronate or estrogen on non-reproductive and reproductive tissues in the long-term dosing of ovariectomized rats. J. Pharmacol. Exp. Ther. 1996, 279, 298-305.

51. Sarahrudi, K.; Thomas, A.; Braunsteiner, T.; Wolf, H.; Vécsei, V.; Aharinejad, S. VEGF serum concentrations in patients with long bone fractures: A comparison between impaired and normal fracture healing. J. Orthop. Res. 2009, 27, 1293-1297.

52. Brighton, C.T.; Lorich, D.G.; Kupcha, R. The pericyte as a possible osteoblast progenitor cell. Clin. Orthop. Relat. Res. 1992, 275, 287-299.

53. Deckers, M.M.; van Bezooijen, R.L.; van der Horst, G.; Hoogendam, J.; van der Bent, C.; Papapoulos, S.E.; Löwik, C.W. Bone morphogenetic proteins stimulate angiogenesis through osteoblast-derived vascular endothelial growth factor A. Endocrinology 2002, 143, 1545-1553. 
54. Maeda, T.; Kawane, T.; Horiuchi, N. Statins augment vascular endothelial growth factor expression in osteoblastic cells via inhibition of protein prenylation. Endocrinology 2003, 144, 681-692.

55. Abe, E.; Yamamoto, M.; Taguchi, Y.; Lecka-Czernik, B.; O’Brien, C.A.; Economides, A.N.; Stahl, N.; Jilka, R.L.; Manolagas, S.C. Essential requirement of BMPs-2/4 for both osteoblast and osteoclast formation in murine bone marrow cultures from adult mice: Antagonism by noggin. J. Bone Miner. Res. 2000, 15, 663-673.

56. Mundy, G.R. Regulation of bone formation by bone morphogenetic proteins and other growth factors. Clin. Orthop. 1996, 324, 24-28.

57. Gutiérrez, O.M.; Mannstadt, M.; Isakova, T.; Rauh-Hain, J.A.; Tamez, H.; Shah, A.; Smith, K.B.A.; Lee, H.; Thadhani, R.; Jüppner, H.; et al. Fibroblast growth factor 23 and mortality among patients undergoing hemodialysis. N. Engl. J. Med. 2008, 359, 584-592.

58. Chen, J.; Shapiro, H.S.; Sodek, J. Development expression of bone sialoprotein mRNA in rat mineralized connective tissues. J. Bone Miner. Res. 1992, 7, 987-997.

59. Meyer, R.A.; Meyer, M.H.; Phieffer, L.S.; Banks, D.M. Delayed union of femoral fractures in older rats: Decreased gene expression. BMC Musculoskelet. Disord. 2001, 2, doi:10.1186/1471-2474-2-2.

60. Hatano, H.; Maruo, A.; Bolander, M.E.; Sarkar, G. Statin stimulates bone morphogenetic protein-2, aggrecan, and type 2 collagen gene expression and proteoglycan synthesis in rat chondrocytes. J. Orthop. Sci. 2003, 8, 842-848.

(C) 2015 by the authors; licensee MDPI, Basel, Switzerland. This article is an open access article distributed under the terms and conditions of the Creative Commons Attribution license (http://creativecommons.org/licenses/by/4.0/). 